\title{
LATTICE-ORDERED INJECTIVE HULLS
}

\author{
BY
}

\author{
STUART A. STEINBERG
}

ABSTRACT. It is well known that the injective hull of a lattice-ordered group ( $l$-group) $M$ can be given a lattice order in a unique way so that it becomes an $l$-group extension of $M$. This is not the case for an arbitrary $f$-module over a partially ordered ring (po-ring). The fact that it is the case for any $l$-group is used extensively to get deep theorems in the theory of $l$-groups. For instance, it is used in the proof of the Hahn-embedding theorem and in the characterization of $x_{a}$-injective $l$-groups.

In this paper we give a necessary and sufficient condition on the injective hull of a torsion-free $f$-module $M$ (over a directed essentially positive po-ring) for it to be made into an $f$-module extension of $M$ (in a unique way). An $f$-module is called an $i$ - $f$-module if its injective hull can be made into an $f$-module extension. The class of torsion-free $i-f$-modules is closed under the formation of products, sums, and Hahn products of strict $f$-modules. Also, an $l$-submodule and a torsionfree homomorphic image of a torsion-free $i$-f-module are $i$-f-modules.

Let $R$ be an $f$-ring with zero right singular ideal whose Boolean algebra of polars is atomic. We show that $R$ is a $q f$-ring (i.e., $R_{R}$ is an $i$-f-module) if and only if each torsion-free $R$-f-module is an $i$-f-module. There are no injectives in the category of torsion-free $R-f$-modules, but there are $\aleph_{\alpha}$-injectives. These may be characterized as the $f$-modules that are injective $R$-modules and $R_{\alpha}$-injective $l$-groups. In addition, each torsion-free $f$-module over $R$ can be embedded in a Hahn product of $l$-simple $Q(R)$-f-modules. We note, too, that a totally ordered domain has an $i-f$-module if and only if it is a right Ore domain.

1. Introduction. Our methods and characterization of torsion-free $i$-f-modules are modelled after Anderson's work on the maximal right quotient ring of an f-ring [1]. Throughout this paper $\mathbf{Z}$ and $\mathbf{Q}$ will denote the totally ordered rings of integers and rational numbers, respectively. If $R$ is a po-ring, then $R_{*}$ will denote the po-ring obtained by freely adjoining $\mathbf{Z}$ to $R$.

We begin by recalling the requisite module theory. All modules will be right modules. An $R$-module $E$ is injective if for every pair of $R$-homomorphisms $f: K \rightarrow E$ and $g: K \rightarrow L$, where $g$ is monic, there exists an $R$-homomorphism $b: L \rightarrow E$ such that $b g=f$. A submodule $N$ of the $R$-module $M$ is an essential submodule (and $M$ is an essential extension of $N$ ) if $N \cap K \neq 0$ for every nonzero submodule $K$ of $M$. Every module $M$ has a maximal essential extension $E=E\left(M_{R}\right)$ which is unique up to an isomorphism over $M . E(M)$ is the smallest injective module containing $M$, and is called the injective bull of $M$. If $F$ is an essential extension of $M$ and

Presented to the Society, February 11, 1971; received by the editors February 10, 1971.

AMS 1970 subject classifications. Primary 06A70; Secondary 16A52, 06A60.

Key words and phrases. $f$-module, lattice-ordered injective hull, relative injectives, torsion-free $f$-module, Hahn embedding theorem. 
it is injective, then $F=E(M)$ ([9] and [10]).

Let $M^{\nabla}$ be the set of essential submodules of $M$. Then $M^{\nabla}$ is a dual ideal in the lattice of submodules of $M$. If $N$ is a submodule of $M$ and $T$ is a subset of $M$, then $(N: T)=\{r \in R: T r \subseteq N\}$ is a right ideal of $R$. If $N \in M^{\nabla}$ and $x \in M$, then $(N: x) \in R^{\nabla}$. For a subset $T$ of $M$ we will sometimes write $r(T)$ for $(0: T)$. In [17] Johnson has defined the singular submodule of $M$ by $Z(M)=\left\{x \in M: r(x) \in R^{\nabla}\right\}$. $M$ is called a torsion-free $R$-module if $Z(M)=0$. Note that when $R$ is a commutative integral domain, $Z(M)$ is just the torsion submodule of $M$.

More generally, if $N$ is a submodule of $M$, then the closure of $N$ in $M$ is defined by $\mathrm{Cl}_{M} N=\left\{x \in M: x D \subseteq N\right.$ for some $\left.D \in R^{\nabla}\right\}$. When no confusion is likely we will write $\mathrm{ClN}$ for $\mathrm{Cl}_{M} N$. ClN is, of course, a submodule of $M$ containing $N$. In fact, $\mathrm{Cl} N / N=Z(M / N)$. $N$ is said to be closed in $M$ if $\mathrm{Cl} N=N$. In general, $\mathrm{ClCl} N$ is the smallest closed submodule of $M$ containing $N$ [13]. The intersection of a family of closed submodules of $M$ is clearly closed. Thus $C_{r}(M)$, the set of closed submodules of $M$, is a complete lattice with greatest lower bound being intersection.

If $Z(M)=0$, then $\mathrm{ClN}$ is the largest essential extension of $N$ contained in $M$. In particular, when $Z(M)=0$, every submodule of $M$ has a unique injective hull contained in $E(M)$. If $K$ is any essential extension of $M$ (and $Z(M)=0$ ), then the map $C_{r}(K) \rightarrow C_{r}(M)$, given by $N \rightarrow N \cap M$, is a lattice isomorphism. The inverse map sends $N$ to its closure in $K$. For proof of these facts see [10].

An $f$-module over the po-ring $R$ is a lattice-ordered $R$-module ( $l$-module) that is embeddable in a product of a family of totally ordered $R$-modules. For the basic properties of $f$-modules see [20].

This paper is based on a portion of the author's dissertation written at the University of Illinois at Urbana-Champaign under the direction of Professor Elliot Weinberg.

2. Torsion-free $f$-modules. A po-ring $R$ is called (right) essentially positive if $D \in R^{\nabla}$ implies $D^{+} R_{*} \in R^{\nabla}$. Since $D^{+} R_{*}=D^{+}-D^{+}+\left(D^{+}-D^{+}\right) R$ is the right ideal of $R$ generated by $D^{+}$, a directed po-ring is essentially positive if and only if each of its essential right ideals contains a directed essential right ideal. In order to characterize the torsion-free $i$-f-modules over $R$ it will be necessary to assume that $R$ is directed and essentially positive. Some examples of essentially positive po-rings are

(1) any totally ordered ring;

(2) any right quotient ring of a torsion-free essentially positive po-ring, in its canonical order;

(3) the $n$-by- $n$ matrix ring over a totally ordered right Ore domain, ordered coordinatewise.

A word about (2) is in order. If $R$ is a ring with $Z\left(R_{R}\right)=0$, then a ring $S$ is 
a right quotient ring of $R$ if $S_{R}$ is an essential extension of $R_{R}$. It is known that $E\left(R_{R}\right)$ can be made into a ring extension of $R$, so $Q(R)=E\left(R_{R}\right)$ is the maximal right quotient ring of $R$ ([17], [22]). Now suppose that $R$ is an essentially positive po-ring, and let $S$ be a right quotient ring of $R$. Then

$$
S^{+}=\left\{s \in S: s D^{+} \subseteq R^{+} \text {for some } D \in R^{\nabla}\right\}
$$

is a partial order of the ring $S$ (Lemma 3.1 implies that $S_{R}$ is a po-module extension of $R_{R}$; but if $a, b \in S^{+}, a D^{+} \subseteq R^{+}$, and $C=\{r \in R: b r \in D\}$, then $a b C^{+} \subseteq$ $a D^{+} \subseteq R^{+}$; also see [1, Theorem 2.3]), called the canonical order of $S$. Note that $S^{+} \cap R$ can contain $R^{+}$properly, and, in fact, $\left(S, S^{+}\right)$is a po-ring extension of $\left(R, R^{+}\right)$if and only if $R$ is essentially semiclosed, i.e. $r D^{+} \subseteq R^{+}$for $D \in R^{\nabla}$ implies $r \in R^{+}$. Let $D$ be an essential right ideal of $S$. Then $D_{R}$ is essential in $S_{R}$. (Suppose that $D \cap X=0$ for some $X_{R} \subseteq S_{R}$. If $0 \neq d \in X S, d=\sum_{i=1}^{n} x_{i} s_{i}$ with $x_{i} s_{i} \neq 0$, then there is an element $t \in R$ such that $d t \neq 0$ and $s_{i} t \in R$. Thus $0 \neq d t \in D \cap X=0$; so $D \cap X S=0$ and $X=0$.) Since $D \cap R \in R^{\nabla},(D \cap R)^{+} R_{*} \epsilon$ $R^{\nabla}$. But then $(D \cap R)^{+} R_{*} S_{*} \in S^{\nabla}$, and since $D^{+} S_{*} \supseteq(D \cap R)^{+} R_{*} S_{*}, D^{+} S_{*} \in S^{\nabla}$. Thus $S$ is essentially positive.

Note, by Proposition 3.2, that a torsion-free right f-ring $R$ (i.e., $R_{R}$ is an $f$ module) is essentially semiclosed. Note also that any torsion-free f-ring is essentially positive [1, Lemma 2.1]. That (3) is true follows easily from (2). For if $D$ is a totally ordered right Ore domain, then $\left(R, R^{+}\right)$is an essentially positive right f-ring with $Z\left(R_{R}\right)=0$ and right quotient ring $\left(D_{n}, D_{n}^{+}\right)$, where $R=$ $\left\{\left[a_{i j}\right] \in D_{n}: a_{i j}=0\right.$ for $\left.j>1\right\}, R^{+}=\left\{\left[a_{i j}\right]: a_{i j} \geq 0\right\}$, and $D_{n}^{+}=\left\{\left[a_{i j}\right] \in D_{n}: a_{i j} \geq 0\right\}$. That $\left(D_{n}, D_{n}^{+}\right)$is essentially positive is actually useless for our purposes, since it has no nontrivial $f$-modules [20].

An $l$-module $M$ is called distributive if the map induced on $M$ by each $r \epsilon$ $R^{+}$is a lattice homomorphism. If $M_{R}$ is distributive, and $x \in M$ and $x R=0$ implies $x=0$, then $M$ is an $f$-module [20].

Lemma 2.1. Let $K$ be a po-module over the essentially positive po-ring $R$, and let $M$ be an essential submodule of $K$.

(a) If $N$ is a convex submodule of $M$, then $\mathrm{Cl}_{K} N$ is a convex submodule of $K$.

(b) If $M$ is a distributive l-module and $N$ is an l-submodule of $M$, then $\mathrm{Cl}_{M} N$ is an l-submodule of $M$.

(c) Suppose that $K$ is a distributive l-module, and $M$ is an l-submodule of $K$. If $N$ is a prime submodule of $M$, then $\mathrm{Cl}_{K} N$ is a prime submodule of $K$.

(d) If $M$ is archimedean and $x$ and $y$ are elements of $K$ such that $n x \leq y$ for all $n \in \mathbf{Z}$, then $x \in Z(K)$. Thus $K$ is archimedean if $M$ is torsion-free and archimedean.

Proof. Suppose that $N$ is a convex submodule of $M$ and $0 \leq x \leq y$ where $x \in K$ 
and $y \in \mathrm{Cl}_{K} N$. Then there exists $D \in R^{\nabla}$ such that $y D \subseteq N$ and $x D \subseteq M$. If $d \epsilon$ $D^{+}$, then $0 \leq x d \leq y d$; so $x D^{+} \subseteq N$. Thus $x \in \mathrm{Cl}_{K} N$, and we have (a).

For (b), suppose $x \in \mathrm{Cl}_{M} N$ and $D \in R^{\nabla}$ such that $x D \subseteq N$. Then $x^{+} d=(x d)^{+}$ $\epsilon N$ for all $d \in D^{+}$. Thus $x^{+} D^{+} \subseteq N$, so $x^{+} \in \mathrm{Cl}_{M} N$.

For (c), suppose that $N$ is a prime submodule of $M$. By (a) and (b), $\mathrm{Cl}_{K} N$ is a convex $l$-submodule of $K$. Suppose that $x$ and $y$ are disjoint elements of $K$, and $x \notin \mathrm{Cl}_{K} N$. There exists $D \in R^{\nabla}$ such that $x D \subseteq M$ and $y D \subseteq M$. Since $x \notin \mathrm{Cl}_{K} N$, there exists $d \in D^{+}$such that $x d \notin N$. If $e \in D^{+}$, then $x d \wedge y e=0$; so ye $\in N$ since $N$ is prime. Thus $y D^{+} \subseteq N$, i.e. $y \in \mathrm{Cl}_{K} N$.

Finally, if $D=(M: x) \cap(M: y)$ where $n x \leq y$ for all $n \in \mathbf{Z}$, then $n x d \leq y d$ for all $d \in D^{+}$. Since $M$ is archimedean, $x d=0$. Therefore, $x D^{+}=0$, and $x \in Z(K)$.

Corollary 2.2. Let $M$ be a distributive l-module over an essentially positive po-ring. Then the closed convex $l$-submodules of $M$ form a complete sublattice of $C_{r}(M)$.

Proof. If $\left\{N_{a}: \alpha \in A\right\}$ is a family of closed convex l-submodules of $M$, then clearly $\bigcap\left\{N_{a}: \alpha \in A\right\}$ is a closed convex l-submodule. By 2.1 , so is $\mathrm{ClCl} \sum N_{\alpha}$.

If $M$ is an $l$-module over a directed po-ring, then whether or not $M$ is an $f-$ module depends only on $P(M)$, the Boolean algebra of polars of $M[20]$. In light of this fact, the following proposition is not surprising. $X^{\perp}$ (or $X^{\perp M}$ ) will denote the polar of a subset $X$ of $M$.

Proposition 2.3. An f-module $M$ over an essentially positive directed po-ring $R$ is torsion-free if and only if each of its (principal) polars is a closed submodule.

Proof. Suppose that $M$ is torsion-free, and let $N$ be a polar of $M$. If $x D \subseteq N$ for some $D \in R^{\nabla}$, then $|x d| \wedge|y|=0$ for all $d \in D^{+}$and for all $y \in N^{\perp}$. So $(|x| \wedge|y|) D^{+}=0$, and hence $x \in N^{\perp \perp}=N$.

The converse is obvious.

Because of 2.2 and 2.3 one might suspect that $P(M)$ is a sublattice of $C_{r}(M)$ when $M$ is torsion-free. However, if $R=\mathbf{Q}$, then each submodule of an l-module is closed, but the sum of two polars need not be a polar.

An $f$-module whose Boolean algebra of polars is atomic will be called irredundant. An irredundant $f$-module $M$ over a directed po-ring is an irredundant subdirect product of totally ordered modules, i.e. $M \subseteq \Pi M_{\alpha}$, where each $M_{\alpha}$ is totally ordered, $M \cap M_{\alpha} \neq 0$, and the set $\left\{M_{\alpha}\right\}$ consists of those homomorphic images of $M$ whose kernels are the maximal polars of $M([14$, p. 40] and [20]).

Corollary 2.4. An irredundant f-module over an essentially positive directed po-ring is torsion-free if and only if it is a subdirect product of totally ordered torsion-free modules. 
Without the irredundancy hypothesis, 2.4 is false (see 2.7). By an essential l-submodule of an l-module we shall mean an $l$-submodule which is also an essential $R$-submodule.

Proposition 2.5. Let $K$ be a torsion-free f-module over the essentially positive directed po-ring $R$, and suppose that $M$ is an essential l-submodule of $K$.

(a) If $N$ is a convex l-submodule of $M$, then $\mathrm{Cl}_{K}\left(N^{\perp M}\right)=\left[\mathrm{Cl}_{K}(N)\right]^{\perp K}$.

(b) If $N$ is a convex l-submodule of $K$, then $N^{\perp K} \cap M=(N \cap M)^{\perp M}$.

Proof. (a) Let $K_{1}=\mathrm{Cl}_{K}\left(N^{\perp M}\right)$ and $K_{2}=\mathrm{Cl}_{K} N$. Recall that $N$ is an essential submodule of $K_{2}$, since $M$ is torsion-free. Thus $K_{1} \cap K_{2}=0$ since $N \cap N^{\perp M}$ $=0$. By $2.1, K_{1}$ and $K_{2}$ are convex $l$-submodules of $K$. Hence $K_{1} \subseteq K_{2}^{\perp K}$. Let $x \in K_{2}^{\perp K}$ and let $D=(M: x)$. Then $x D \subseteq N^{\perp M} \subseteq K_{1}$. Thus, since $D$ is an essential right ideal and $K_{1}$ is closed in $K, x \in K_{1}$. So $K_{1}=K_{2}^{\perp K}$.

(b) It is clear that $N^{\perp K} \cap M \subseteq(N \cap M)^{\perp M}$. Let $x$ be a positive element of $(N \cap M)^{\perp M}$, and let $y$ be a positive element of $N$. Since $N$ is an essential extension of $N \cap M, y D \subseteq N \cap M$ for some essential right ideal $D$. If $d \in D^{+}$, then $x \wedge$ $y d=0$, so $(x \wedge y) D^{+}=0$. Since $M$ is torsion-free, $x \in N^{\perp K}$. Thus $N^{\perp K} \cap M=$ $(N \cap M)^{\perp M}$.

Corollary 2.6. Let $K$ be a torsion-free f-module over the essentially positive directed po-ring $R$, and suppose that $M$ is an essential l-submodule of $K$. Then the map $N \rightarrow \mathrm{Cl}_{K} N$ is an isomorphism between the Boolean algebras of polars of $M$ and $K$. Its inverse is the map $N \rightarrow N \cap M$.

Proof. We already know that these correspondences are one-to-one between $C_{r}(M)$ and $C_{r}(K)$. By 2.5 they take polars to polars. In fact, if $N \in P(K)$, then $N=\mathrm{Cl}_{K}(N \cap M)=\mathrm{Cl}_{K}\left(N^{\perp K \perp K} \cap M\right)=\mathrm{Cl}_{K}\left[\left(N^{\perp K} \cap M\right)^{\perp M}\right]$. Thus the map $N \rightarrow$ $\mathrm{Cl}_{K} N$ between $P(M)$ and $P(K)$ is an order isomorphism that is onto. Hence it is an isomorphism of Boolean algebras.

A qf-ring is an $f$-ring $R$ whose maximal right quotient ring $Q$ is an $f$-ring extension [1]. If $R$ is a $q$-ring with $Z(R)=0$, then $Q=E\left(R_{R}\right)$ is a (strongly) regular self-injective ring.

Proposition 2.7. Let $R$ be a semiprime (right) qf-ring with maximal right quotient ring $Q$. The following are equivalent:

(a) $R_{R}$ is a subdirect product of totally ordered torsion-free modules.

(b) $Q_{R}$ is a subdirect product of totally ordered torsion-free modules.

(c) $Q_{Q}$ is a subdirect product of totally ordered torsion-free modules.

(d) The Boolean algebra of polars of $Q$ is atomic.

(e) The Boolean algebra of polars of $R$ is atomic.

(f) $Q$ is the direct product of a family of totally ordered division rings. 
Proof. (a) implies (b). Let $\left\{N_{a}: \alpha \in A\right\}$ be a collection of closed prime submodules of $R_{R}$ whose intersection is zero, and let $E_{a}=E\left(\left(N_{a}\right)_{R}\right) \subseteq Q$. By 2.1 each $E_{\alpha}$ is a closed prime submodule of $Q_{R}$. Since $C_{r}(R)$ and $C_{r}(Q)$ are isomorphic via the correspondence $N \rightarrow E\left(N_{R}\right), \bigcap\left\{E_{\alpha}: \alpha \in A\right\}=0$.

That (b) implies (c) follows from the fact that every closed submodule of $Q_{R}$ is a right ideal of $Q[10, \mathrm{p} .70]$.

(c) implies (d). Let $\left\{E_{a}: \alpha \in A\right\}$ be a collection of closed prime submodules of $Q_{Q}$ whose intersection is zero. Since $Q_{Q}$ is injective, $Q=E_{\alpha} \oplus F_{a}$ as $Q$ modules. Since $Q$ is a regular $f$-ring, each right ideal is an $l$-ideal, so the direct sum is a sum of $f$-rings. Thus each $F_{a}$ is a totally ordered division ring. The projections onto the $F_{a}$ induce an isomorphism of $Q$ into the product of the $F_{a}$ whose image contains the direct sum. Thus $P(Q)$ is atomic.

Finally, (d) and (e) are equivalent by 2.6 , (e) implies (a) by 2.4 , and (d) is equivalent to (f) by [10, p. 117].

It is, of course, not always the case that $P(R)$ is atomic. For instance, it is known that $C([0,1])$, the f-ring of real-valued continuous functions defined on the unit interval, has no maximal polars. We do, however, have the following positive results:

Proposition 2.8. Let $R$ be a directed po-ring which has the property that a right ideal is essential if and only if it contains a positive regular element. If $M$ is a torsion-free f-module over $R$, then every minimal prime submodule of $M$ is closed. Thus $M$ is a subdirect product of totally ordered torsion-free f-modules.

Proof. First note that $R$ is essentially positive. Suppose that $N$ is a minimal prime submodule of $M$. By $2.1, \mathrm{Cl} N$ is a convex $l$-submodule of $M$. If $N \subsetneq$ $\mathrm{Cl} N$, there exists a positive element $x \in(\mathrm{Cl} N) \backslash N$. By hypothesis $(N: x)$ contains a positive regular element $d$. Since $N$ is a minimal prime subgroup [20], there exists $y \in M^{+} \backslash N$ such that $y \wedge x d=0$ [16]. Therefore $(y \wedge x) d=y d \wedge x d=0$. Since $M$ is torsion-free and $d R$ is an essential right ideal of $R, x \wedge y=0$. Thus $x \in N$, since $N$ is prime.

A semiprime right Goldie ring can be characterized as a ring $R$ that has a classical right quotient ring which is semisimple and artinian ([12] and [10]). $R$ satisfies and, in fact, is characterized by the following condition: A right ideal $I$ of $R$ is essential if and only if it contains a regular element. If $R$ is also an $f$-ring, then, since it has no nilpotent elements, it is of the following form: There is a family of totally ordered right Ore domains $\left\{R_{i}: i=1, \ldots, n\right\}$ with totally ordered quotient division rings $\left\{D_{i}: i=1, \cdots, n\right\}$ such that $R$ is (isomorphic to) an l-subring of $D_{1} \oplus \ldots \oplus D_{n}$ containing $R_{1} \oplus \ldots \oplus R_{n}$ (see [10] and [1]).

The ring $R$ of 2.8 is a semiprime right Goldie ring. It need not be an f-ring, however, but, for example, need only be directed and have the property that the 
square of every element is positive. We suspect that $R$ cannot have any nilpotent elements.

A module $M$ over the semiprime ring $R$ is called I-torsion-free [18] if $N J=0$ for $0 \neq N_{R} \subseteq M$ and some ideal $J$ of $R$ implies $J K=0$ for some nonzero ideal $K$. Note that if $M$ is torsion-free, then it is $l$-torsion-free. For if $N J=0$, then $J_{R}$ essential in $R$ implies $J=0$, while $J \cap K=0$ for $0 \neq K_{R} \subseteq R$ implies $K J \subseteq J$ $\cap K=0$, and hence $J K=0$.

Theorem 2.9. Let $R$ be a torsion-free right qf-ring. Then every torsion-free fmodule over $R$ is a subdirect product of totally ordered torsion-free modules if and only if the Boolean algebra of polars of $R$ is atomic.

Proof. Suppose that $P(R)$ is atomic. By 2.7, $Q(R)=\Pi D_{a}$, where $D_{a}(\alpha \in A)$ is a totally ordered division ring. Let $R_{\alpha}=\operatorname{image}\left(R \rightarrow D_{\alpha}\right)$. Then $R$ is an irredundant subdirect product of the $R_{a}: R \subseteq \Pi R_{\alpha} \subseteq \Pi D_{\alpha}$. Since $Q\left(\Pi R_{\alpha}\right)=\Pi D_{a}, D_{\alpha}=$ $Q\left(R_{\alpha}\right)[22,2.2]$. But then $R_{\alpha}$ is a right Ore domain $[1,5.2]$.

Let $M$ be a torsion-free $f$-module over $R$, let $N_{a}=\left\{x \in M: x\left(R \cap R_{\alpha}\right)=0\right\}$, and let $P_{a}=\operatorname{kernel}\left(R \rightarrow R_{\alpha}\right)$. By $[18,3.7], M P_{\alpha} \subseteq N_{\alpha}, M_{\alpha}=M / N_{a}$ is an l-torsionfree $R$-module ( $R_{\alpha}$-module), and $M$ is a subdirect product of the $M_{\alpha}$ (as $R$-modules). It is easily seen that $N_{a}$ is a convex $l$-submodule of $M$, so the subdirect product is one of $f$-modules. Clearly, $M_{a}$ is an f-module over $R_{a}$.

Let $E_{a}$ be the $R_{a}$-injective hull of $M_{a}$. Then $E_{a}$ is the $R$-injective hull of $M_{a}$ and $E\left(M_{R}\right)=E=\Pi E_{a}[18,4.1]$. Thus each $E_{a}$ is a torsion-free $R$-module. But $R_{a}$ $=R / P_{a}$ and $M_{a} P_{a}=0$, so $M_{a}$ is a torsion-free $R_{a}$-module [13, Lemma 3.4].

Let $N_{a}$ be a minimal prime $R$-submodule of $M_{a}$. Then $N_{a}$ is a minimal prime $R_{a}$-submodule of $M_{\alpha}$, and so, by 2.8 , is a closed $R_{\alpha}$-submodule. Suppose that $x T$ $\subseteq N_{a}$ for some $x \in M_{\alpha}$ and some essential right ideal $T$ of $R$. If $T \subseteq P_{\alpha}$, then $P_{\alpha}$ $\in R^{\nabla}$. This contradicts the fact that $R_{a}$ is a torsion-free $R$-module (2.4). Thus $T$ $\subsetneq P_{\alpha}$, and, since every nonzero right ideal of $R_{\alpha}$ is essential, $x \in N_{\alpha}$. So $N_{\alpha}$ is a closed $R$-module. Therefore $M_{\alpha}$, and hence $M$, is a subdirect product of totally ordered torsion-free $R$-modules.

The converse is given by the equivalence of (a) and (e) of 2.7.

Proposition 2.10. Let $M$ be an essential l-submodule of the f-module $K_{R}$. If $R$ is directed, or if it is essentially positive and $M$ is torsion-free, then any weak order unit of $M$ is a weak order unit of $K$. Thus, $K$ is totally ordered if and only if $M$ is.

Proof. Suppose that $x \in K$ with $x^{\perp} \neq 0$. If $R$ is directed, then $x^{\perp}$ is a submodule of $K$, so $x^{\perp} \cap M \neq 0$. Suppose $M$ is torsion-free and $R$ is essentially positive, and let $0 \neq y \in x^{\perp}$. There exists $d \in(M: y)^{+}$such that $y d \neq 0$. So $y d \epsilon$ $x^{\perp} \cap M$. Thus, in either case, $x^{\perp} \cap M \neq 0$, and a weak order unit of $M$ is a weak 
order unit of $K$. If $M$ is totally ordered and $x$ is a nonzero element of $K$, then any positive nonzero element of $x^{\perp} \cap M$ is a weak order unit of $M$. So $x^{\perp} \cap M=0$. If $R$ is directed this says that $x^{+}=0$, since $x^{\perp}$ is a submodule of $K$. If $R$ is essentially positive, $M$ is torsion-free, and $0 \neq y \in x^{\perp}$, then $0 \neq y d \in x^{\perp} \cap M$ for some $d \in(M: y)^{+}$. Thus $x^{\perp}=0$. So $K$ is totally ordered in both cases.

An element $g$ in the $f$-module $M$ (over the directed po-ring $R$ ) is called basic [4] if $C_{R}(g)$, the convex $l$-submodule generated by $g$, is totally ordered. This is equivalent to saying that $C(g)\left(=C_{Z}(g)\right)$ is totally ordered. A subset $X$ of $M$ is a basis of $M$ if $X$ is a maximal set of disjoint elements and each element of $X$ is basic.

Proposition 2.11. Let $R$ be a directed po-ring, and let $M$ be an essential lsubmodule of the f-module $K_{R}$. Then $M$ bas a basis of cardinality $u$ if and only if $K$ bas a basis of cardinality $u$.

Proof. If $x$ and $y$ are two elements of $K$ such that $0 \neq x \in y^{\perp}$, and if $r \in R_{*}$ is such that $0 \neq x r \in M$, then $x r \in y^{\perp}$, since every polar of $K$ is a submodule. Let $X=\left\{x_{a}: \alpha \in A\right\}$ be a maximal set of disjoint elements of $K$. There is a subset $\left\{r_{a}\right.$ : $\alpha \in A\}$ of $R_{*}$ such that $Y=\left\{\left|x_{a} r_{a}\right|: \alpha \in A\right\}$ is a set of disjoint elements of $M$. If $X$ is now a basis of $K$, then, clearly, each element of $Y$ is basic. Suppose that $y$ is a nonzero element of $M$ such that $y \wedge\left|x_{\alpha} r_{a}\right|=0$ for all $\alpha \in A$. Let $\alpha$ be an element in $A$ such that $y \wedge x_{a}>0$. Then $y \wedge x_{a} \wedge\left|x_{a} r_{a}\right|=0$, contradicting the fact that $x_{a}$ is basic. So $Y$ is a basis of $M$.

On the other hand, if $Y=\left\{y_{a}: \alpha \in A\right\}$ is a maximal set of disjoint elements of $M$, and if $0 \neq x \in K$ is such that $x \wedge y_{\alpha}=0$ for all $\alpha \in A$, then $Y \cup\{|x r|\}$ is a disjoint set in $M$ for some $r \in R_{*}$. Thus $x$ does not exist. So $Y$ is a maximal set of disjoint elements of $K$. Since the convex $l$-submodule of $K$ generated by an element of $M$ is totally ordered exactly when the convex $l$-submodule of $M$ generated by it is totally ordered, $Y$ is a basis of $M$ if and only if it is a basis of $K$.

For an $f$-module $M_{R}, \Gamma_{R}(M)$ will denote the rooted po-set of $R$-values of $M$ [20]. It is known [7] that an f-module $M$ has a finite basis with $n$ elements if and only if $\Gamma_{Z}(M)$ has exactly $n$ roots. Since there is a one-to-one correspondence between the roots of $\Gamma_{R}$ and the minimal prime submodules of $M$, and since the sets of minimal prime subgroups and submodules coincide, $M$ has a basis containing $n$ elements exactly when $\Gamma_{R}$ has $n$ roots. This fact gives the following corollary.

Corollary 2.12. Let $M$ be an i-f-module over the directed po-ring R. Then. $\Gamma_{R}(M)$ bas exactly $n$ roots if and only if $\Gamma_{R}(E)$ bas exactly $n$ roots.

Let $M$ be an l-group, and let $d(M)$ be its $\mathrm{Z}$-injective hull. There is a lattice isomorphism between the lattice of convex $l$-subgroups of $M, \mathcal{L}(M)$, and the lattice of convex l-subgroups of $d(M)$ given by: $N \in \mathcal{L}(M)$ corresponds to the convex 
l-subgroup of $d(M)$ generated by $N$, and $N \in \mathscr{L}(d(M))$ corresponds to $N \cap M$. With respect to this correspondence, the value sets of $M$ and $d(M)$ are isomorphic.

That the $R$-value sets of $M$ and $E\left(M_{R}\right)$ are not always isomorphic for an (totally ordered torsion-free) $i$-f-module $M_{R}$ is shown by the following example.

Example 2.13. Let $R=\mathbf{Q}[[x]]$ be the formal power series ring with coefficients in $\mathbf{Q}$ and exponents in $\mathbf{Z}^{+}$. Order $R$ lexicographically with the constant term dominating. Thus $R=\left\{\sum_{i=0}^{\infty} a_{i} x^{i}: a_{i} \in \mathbf{Q}\right\}$ and $R^{+}=\left\{\sum_{i=n}^{\infty} a_{i} x^{i}: a_{n}>0\right\} \cup\{0\}$. The units of $R$ are the elements with nonzero constant term. Every element of $R$ is of the form $x^{k} u, u$ a unit or zero. The quotient field $F$ of $R$ is $\left\{\sum_{i=-n}^{\infty} a_{i} x^{i}: n \geq 0, a_{i} \epsilon\right.$. Q\}. It is a totally ordered field if its positive cone is defined by $F^{+}=\left\{\sum_{i=-n}^{\infty} a_{i} x^{i}\right.$ : $\left.a_{-n}>0\right\}$.

Let $M=R_{R}$. Then $E\left(M_{R}\right)=F_{R}$, so $M$ is an $i$-f-module. The convex $l$-submodules of $M$ are

$$
R \supseteq x R \supseteq x^{2} R \supseteq \cdots,
$$

and the convex $l$-submodules of $E$ are

$$
F \supseteq \cdots \supseteq x^{-2} R \supseteq x^{-1} R \supseteq R \supseteq x R \supseteq x^{2} R \supseteq \cdots
$$

Thus $\Gamma_{R}(M) \cong \mathrm{Z}^{+}$and $\Gamma_{R}(E) \cong \mathrm{Z}$.

3. i-f-modules. In this section we show that part of Anderson's characterization of a unital $q f$-ring [1] characterizes the torsion-free $i$-f-modules over an essentially positive directed po-ring. Using this characterization we show that a totally ordered domain has torsion-free $i$-f-modules if and only if it is a right Ore domain. We also show that every torsion-free $f$-module over a torsion-free irredundant $f$-ring $R$ is an $i$-f-module if and only if $R$ is a $q f$-ring. In addition, we examine some properties of the class of torsion-free $i$-f-modules, and we show that the $i$-f-property is a local property over a right noetherean ring.

Let $M$ be a po-module over the po-ring $R$, and let $N$ be an $R$-module containing $M$. Define

$$
N^{+}=\left\{x \in N: x D^{+} \subseteq M^{+} \text {for some } D \in R^{\nabla}\right\}
$$

Notice that if $R$ contains an essential right ideal $D$ for which $D^{+}=0$, then $N^{+}=$ $N$. When $R=\mathrm{Z}, N^{+}$consists of those elements $x$ of $N$ such that $n x \in M^{+}$ for some positive integer $n$.

Lemma 3.1.

(a) $N^{+}+N^{+} \subseteq N^{+}$.

(b) $N^{+} R^{+} \subseteq \bar{N}^{+}$.

(c) If $R$ is essentially positive, then $N^{+} \cap-N^{+}=Z\left(N_{R}\right)$.

(d) If $R$ is essentially positive and if $M$ is a distributive l-module, then $M^{+}$ $\subseteq N^{+} \cap M=\left\{x \in M: x^{-} \in Z\left(M_{R}\right)\right\}$. 
Proof. The first statement is an immediate consequence of the fact that $R^{\nabla}$ is closed under intersection.

For (b), take $x \in N^{+}, a \in R^{+}$, and $D \in R \nabla$ such that $x D^{+} \subseteq M^{+}$. Let $I=$ $(D: a)$. Then $I \in R^{\nabla}$, and $(x a) I^{+}=x\left(a I^{+}\right) \subseteq x D^{+} \subseteq M^{+}$. Hence $x a \in N^{+}$.

If $x \in Z(N)$, then $x D=0$ for some $D \in R \nabla$; so, clearly, $x,-x \in N^{+}$. Conversely, if $x,-x \in N^{+}$, then $x D^{+},-x F^{+} \subseteq M^{+}$for some $D, F \in R^{\nabla}$. Therefore, $x(D \cap F)^{+} \subseteq M^{+} \cap-M^{+}=0, x \in Z(N)$, and $N^{+} \cap-N^{+}=Z(N)$.

Finally, if $x \in M$ with $x^{-} \in Z(M)$, then $x D^{+}=\left(x^{+}-x^{-}\right) D^{+}=x^{+} D^{+} \subseteq M^{+}$for some $D \in R^{\nabla}$. Thus $x \in N^{+} \cap M$. On the other hand, if $x \in N^{+} \cap M$ and $x D^{+} \subseteq M^{+}$ for some $D \in R^{\nabla}$, then $x^{-} d=(x d)^{-}=0$ for all $d \in D^{+}$. So $x^{-} \in Z(M)$.

Proposition 3.2. Let $M$ be a distributive l-module over the essentially positive po-ring $R$, and let $N$ be a torsion-free $R$-module containing $M$. Then $N^{+}=$ $\left\{x \in N: x D^{+} \subseteq M^{+}\right.$for some $\left.D \in R^{\nabla}\right\}$ is a partial order on $N$, and $\left(N, N^{+}\right)$is a pomodule extension of $\left(M, M^{+}\right)$. If $N$ is an essential extension of $M$, then

(a) $N$ is semiclosed.

(b) The greatest lower bound (least upper bound) of two elements of $M$ is also their greatest lower bound (least upper bound) in $N$.

(c) $N^{+}$is the largest partial order $P$ of $N$ for which $(N, P)$ is a po-module extension of $\left(M, M^{+}\right)$.

(d) $N^{+}=\left\{x \in N: x(N: x)^{+} \subseteq M^{+}\right\}$.

Proof. By 3.1, $\left(N, N^{+}\right)$is a po-module extension of $\left(M, M^{+}\right)$. Suppose that $N$ is an essential extension of $M$. Let $x \in M$ and $y \in N$ with $y \geq x, 0$. Since $N$ is an essential extension of $M,(M: y) \in R^{\nabla}$. If $d \in(M: y)^{+}$, then $y d \geq(x d)^{+}=x^{+} d$. Therefore, $\left(y-x^{+}\right)(M: y)^{+} \subseteq M^{+}$, so $y-x^{+} \in N^{+}$. Thus $x^{+}$is the least upper bound of $x$ and 0 in $N$, and we have (b). A similar argument gives (a).

Suppose that $P$ is a partial order on $N$ for which $P \cap M=M^{+}$and $P R^{+} \subseteq P$. If $y \in P$, then $y(M: y)^{+} \subseteq P R^{+} \cap M \subseteq P \cap M=M^{+}$. Thus $y \in N^{+}$and $P \subseteq N^{+}$. Therefore $(c)$ is true.

The last statement is obvious.

Proposition 3.3. Let $M$ be a distributive torsion-free l-module over the essentially positive po-ring $R$, and let $N_{R}$ be an essential extension of $M_{R}$. If $(N, P)$ is a distributive l-module extension of $\left(M, M^{+}\right)$, then $P=N^{+}$.

Proof. By 3.2(c) we only have to show that $N^{+} \subseteq P$. Take $x \in N^{+}$and let $D$ $=(M: x) \cap\left(M: x^{+}\right)$. (All lattice operations are with respect to $P$.) If $x \notin P$, then $x^{-} \neq 0$, and $(x d)^{-}=x^{-} d \neq 0$ for some $d \in D^{+}$, i.e. $x d \in M \cap N^{+}=M^{+}$and $(x d)^{-} \not$ 0 . Thus $x \in P$, and $N^{+} \subseteq P$.

Notice that 3.3 says that $N^{+}$is the only partial order $P$ of $N$ for which $(N, P)$ can be a distributive $l$-module extension of $\left(M, M^{+}\right)$. 
Theorem 3.4. Let $M$ be a torsion-free right f-module over the essentially positive directed po-ring $R$. Then the injective bull $E$ of $M$ is an f-module extension of $M$ if and only if for all $x \in E$ and, for all $d_{1}, d_{2} \in R^{+}$for which $x d_{i}$ $\in M$,

$$
\left(x d_{1}\right)^{+} \wedge\left(x d_{2}\right)^{-}=0
$$

When this is the case the lattice order of $E$ is uniquely determined by that of $M$.

Proof. First note that this condition is equivalent to: If $\phi$ is a homomorphism of $M$ onto a totally ordered $R$-module and $x \in E$, then $\phi\left(x(M: x)^{+}\right) \subseteq \phi(M)^{+}$or $-\phi\left(x(M: x)^{\dagger}\right) \subseteq \phi(M)^{+}$. For if this latter condition is satisfied and $d_{1}, d_{2} \epsilon$ $(M: x)^{+}$, then

$$
\phi\left[\left(x d_{1}\right)^{+} \wedge\left(x d_{2}\right)^{-}\right]=\phi\left(x d_{1}\right)^{+} \wedge \phi\left(x d_{2}\right)^{-}=0
$$

for arbitrary $\phi$. Therefore, $\left(x d_{1}\right)^{+} \wedge\left(x d_{2}\right)^{-}=0$, since $M$ is a subdirect product of totally ordered $R$-modules. Conversely, suppose that the condition in the theorem is satisfied and $\phi\left(x d_{1}\right)>0, \phi\left(x d_{2}\right)<0$. Then $\phi\left[\left(x d_{1}\right)^{+} \wedge\left(x d_{2}\right)^{-}\right]=\phi\left(x d_{1}\right)^{+} \wedge$ $\phi\left(x d_{2}\right)^{-}=\phi\left(x d_{1}\right) \wedge-\phi\left(x d_{2}\right)>0$, which contradicts the fact that $\left(x d_{1}\right)^{+} \wedge\left(x d_{2}\right)^{-}$ $=0$.

If $E$ is an $f$-module extension of $M$, then clearly $\left(x d_{1}\right)^{+} \wedge\left(x d_{2}\right)^{-}=x^{+} d_{1} \wedge$ $x^{-} d_{2}=0$. Conversely, suppose that the condition holds. By 3.2, $\left(E, E^{+}\right)$is a pomodule extension of $\left(M, M^{\dagger}\right)$. Let $x \in E$, and consider the correspondence $b$ : $(M: x)^{+} R \rightarrow E$ given by $\sum_{i=1}^{n} d_{i} r_{i} \rightarrow \Sigma_{i=1}^{n}\left(x d_{i}\right)^{+}{ }_{r_{i}}$. Suppose that $\Sigma d_{i^{r}{ }_{i}}=0$. Let $\phi: M$ $\rightarrow \phi(M)$ be any homomorphism onto a totally ordered $R$-module. If $\phi\left(x(M: r)^{\dagger}\right) \subseteq$ $\phi(M)^{+}$, then

$$
0=\phi\left(\sum x d_{i} r_{i}\right)=\sum \phi\left(x d_{i}\right)^{+} r_{i}=\phi\left[\sum\left(x d_{i}{ }^{+}{ }^{+} r_{i}\right]\right.
$$

and similarly, if $-\phi\left(x(M: x)^{+}\right) \subseteq \phi(M)^{+}$, then

$$
\phi\left[\sum\left(x d_{i}\right)^{+} r_{i}\right]=\sum \phi\left(x d_{i}\right)^{+} r_{i}=0 .
$$

Since $M$ is a subdirect product of totally ordered $R$-modules, $\Sigma\left(x d_{i}\right)^{+} r_{i}=0$, and thus $b$ is a well-defined function. Clearly, it is an $R$-homomorphism. Since $E$ is an injective $R$-module, $b$ can be extended to an $R$-homomorphism $g$ defined on $R_{*}$.

Let $y=g(1)$. Then $b(d r)=g(d r)=y d r$ for all $d \in(M: x)^{+}$and $r \in R$, so $y d r=$ $(x d)^{+}$r. Since $Z(E)=0, y d=(x d)^{+}$for all $d \in(M: x)^{+}$. We claim that $y=x^{+}$. Since $y(M: x)^{+} \subseteq M^{+}, y$ is in $E^{+}$. Also, $(y-x) d=(x d)^{+}-x d \geq 0$ for all $d \epsilon$ $(M: x)^{+}$, so $y-x \in E^{+}$. Suppose that $z \in E$ with $z \geq\{0, x\}$. If $0 \leq d \in(M: x) \cap(M: z)$, then $z d \geq(x d)^{+}=y d$, so $z-y \in E^{+}$. Therefore $y=x^{+}$, and $E$ is an $l$-module. Note that $x^{+} d=(x d)^{+}$for all $d \in(M: x)^{+}$. 
All that remains is to show that $E$ is an $f$-module. Take $x \in E, a \in R^{+}$, and let $C=\{b \in R: a b \in(M: x)\}$. Then $C \in R^{\nabla}$, and if $b \in C^{+},\left(x^{+} a\right) b=(x a b)^{+}=$ $(x a)^{+} b$. Thus $\left[x^{+} a-(x a)^{+}\right] C^{+}=0$, and hence $x^{+} a=(x a)^{+}$. Therefore $E$ is a distributive $l$-module, and, hence it is an $f$-module.

Note that without the assumption that $R$ is directed, Theorem 3.4 becomes

$E$ is a distributive l-module extension of $M$ if and only if $\left(x d_{1}\right)^{+} \wedge\left(x d_{2}\right)^{-}=$ 0 for all $x \in E$ and for all $d_{1}, d_{2} \in R^{+}$such that $x d_{i} \in M$.

Corollary 3.5. Let $M$ be a torsion-free f-module over the po-ring $R$. Then $M$ is an i-f-module if either

(a) $R$ is commutative, essentially positive, and directed, or

(b) $R$ is an f-ring and a semiprime right Goldie ring.

Proof. (a) If $x \in E(M)$ and $d_{1}, d_{2}, d \in(M: x)^{+}$, then

$$
\left[\left(x d_{1}\right)^{+} \wedge\left(x d_{2}\right)^{-}\right] d=\left(x d_{1} d\right)^{+} \wedge\left(x d_{2} d\right)^{-}=(x d)^{+} d_{1} \wedge(x d)^{-} d_{2}=0 .
$$

Thus $\left[\left(x d_{1}\right)^{+} \wedge\left(x d_{2}\right)^{-}\right](M: x)^{+}=0$, so $\left(x d_{1}\right)^{+} \wedge\left(x d_{2}\right)^{-}=0$.

(b) Let $x \in E(M), d_{1}, d_{2} \in(M: x)^{+}$, and $d \in(M: x)^{+}$with $d$ regular. There are elements $a, b, c, e \in R^{+}$, with $a$ and $c$ regular, such that $d_{1} d a=d b$ and $d_{2} d c=$ de. Therefore,

$$
\left(x d_{1} d a\right)^{+} \wedge\left(x d_{2} d c\right)^{-}=(x d b)^{+} \wedge(x d e)^{-}=(x d)^{+} b \wedge(x d)^{-} e=0
$$

so $\left(x d_{1}\right)^{+} d a \wedge\left(x d_{2}\right)^{-} d c=0$. Let $s$ and $t$ be positive regular elements of $R$ such that $a s=c t$. Then

$$
\left[\left(x d_{1}\right)^{+} \wedge\left(x d_{2}\right)^{-}\right] \text {das }=\left(x d_{1}\right)^{+} d a s \wedge\left(x d_{2}\right)^{-} d c t=0 .
$$

But das is regular. Therefore das $R$ is an essential right ideal of $R$, and $\left(x d_{1}\right)^{+}$ $\wedge\left(x d_{2}\right)^{-}=0$.

Note that any commutative, semiprime, directed po-ring, in which the square of every element is positive, is an example of a po-ring satisfying the conditions of 3.5(a). An archimedean semiprime f-ring is, of course, such a po-ring.

The equivalence of (1) and (2) in the next corollary is a generalization of the fact that a semiprime $f$-ring $R$ with the maximum condition on polars (i.e., $P(R)$ is finite) is a right $q$-ring if and only if it is a right Goldie ring [1, Theorem 6.1].

Corollary 3.6. The following statements are equivalent for an irredundant torsion-free f-ring $R$.

(1) $R$ is a qf-ring.

(2) Each component of the irredundant representation of $R$ is a totally ordered right Ore domain.

(3) Every torsion-free f-module over $R$ is an i-f-module. 
Proof. That (1) implies (2) has already been observed in the proof of 2.9, and that (3) implies (1) follows from the fact that $Q(=E(R))$ is an $f$-module extension of $R_{R}$ if and only if it is an $f$-ring extension of $R$.

Let $M$ be a torsion-free $f$-module over $R$. Assuming (2), we have (see the proof of 2.9): $R \subseteq \Pi R_{\alpha}$ and $M \subseteq \Pi M_{\alpha} \subseteq \Pi E_{a}=E(M)$, where $R_{a}$ is a totally ordered right Ore domain, and $E_{a}$ is the $R_{a^{-}}\left(R\right.$-)injective hull of the torsion-free $R_{a^{-}}$$(R-) f$-module $M_{a}$. By 3.5, each $E_{\alpha}$ is an $f$-module extension of $M_{\alpha}$ (over $R_{\alpha}$, hence over $R$ ). Thus $E(M)$ is an $f$-module extension of $M$, and (2) implies (3).

An interesting example of a torsion-free $i$-f-module may be obtained as follows. Let $R$ be a semiprime right $q$-ring with maximal right quotient ring $Q$. Suppose that $I$ is an $l$-submodule of $Q_{R}$, and let $I^{\prime}=E\left(I_{R}\right) \subseteq Q$. Then $I^{\prime}=e Q$ for some idempotent $e$ of $Q$. Consequently, $S=\operatorname{Hom}_{R}\left(I_{R}, I_{R}\right) \cong\{q \in Q e: q I \subseteq I\}$, and $T=$ $\operatorname{Hom}_{R}\left(I_{R}^{\prime}, I_{R}^{\prime}\right) \cong e Q$. Thus $S$ and $T$ can be made into $f$-rings in a natural way. Now $S_{S}$ is an essential submodule of $T_{S}[10, \mathrm{p} .97]$, and $S^{I}$ is a torsion-free $f-$ module [21, Theorem 3.25]. If $s^{S}$ is essential in $s^{T}$, then $s^{I}$ is an $i$-f-module.

There are $i-f$-modules that are not torsion-free. For instance, over a quasiFrobenius $f$-ring $R$ each unital module can be made into an $f$-module. If $R$ is totally ordered, but not a division ring, then it has no torsion-free modules $[21, \mathrm{p}$. 114]. It is not hard to see that if $M$ is any $i$-f-module over a directed essentially positive po-ring, then $M / Z_{2}(M)$ is a torsion-free $i$-f-module, where $Z_{2}(M)=\mathrm{ClCl} 0$ is the torsion submodule of $M$.

We now present an example of a totally ordered, archimedean, torsion-free $f$ module, over a unital essentially positive $l$-ring, that is not an $i$-f-module, but whose injective hull is an $l$-module extension.

Example 3.7. Let $R=\left\{\left(\begin{array}{ll}a & 0 \\ b & c\end{array}\right): a, b, c \in \mathbf{Q}\right\}$, and let $R^{+}=\left\{\left(\begin{array}{ll}a & 0 \\ b & c\end{array}\right): a, b, c \in \mathbf{Q}^{+}\right\}$. Then $R$ is an essentially positive unital l-ring. Let $M=\left\{\left(\begin{array}{ll}0 & 0 \\ a & 0\end{array}\right): a \in \mathbf{Q}\right\}$, and let $M^{+}=\left\{\left(\begin{array}{ll}0 & 0 \\ a & 0\end{array}\right): a \in \mathbf{Q}^{+}\right\}$. Then $M_{R}$ is a simple $R$-module and a totally ordered torsionfree $f$-module. But $E\left(M_{R}\right)=\left\{\left(\begin{array}{ll}0 & 0 \\ a & b\end{array}\right): a, b \in \mathbf{Q}\right\}$ cannot be made into an f-module extension of $M$ (use 3.4).

More generally, if $R$ is any essentially positive sub-po-ring of the canonically ordered matrix ring $D_{n}(n>1, D$ a totally ordered division ring $)$, such that $Q(R)=D_{n}$, then 3.12 implies that $R$ has no torsion-free $i$-f-module $\left(D_{n}\right.$ has no nontrivial $f$-modules). Any $l$-subring $R$ of $D_{n}$ with $Q(R)=D_{n}$ is essentially positive.

Proposition 3.8. Let $M$ be a torsion-free i-f-module over an essentially positive po-ring $R$.

(a) Every l-submodule of $M$ is an i-f-module.

(b) If $N$ is a closed convex l-submodule of $M$, then $M / N$ is an i-f-module.

Proof. Let $E=E\left(M_{R}\right)$. If $N$ is an l-submodule of $M$, then $E(N)=\mathrm{Cl}_{E} N$ is an $l$-submodule of $E$ by $2.1(\mathrm{~b})$. Thus $N$ is an $i$-f-module. If $N$ is a closed convex 
l-submodule of $M$, then $E_{1}=E(N)$ is a convex l-submodule of $E$ by 2.1 , and $N=$ $E_{1} \cap M$. Therefore, $M / N \rightarrow E / E_{1}$ is a mononomorphism of $f$-modules. Since $E=$ $E_{1} \oplus E_{2}$ as $R$-modules, $E / E_{1}$ is an injective $R$-module. But $M / N$ is an $l$-submodule of $E / E_{1}$, so $M / N$ is an $i$-f-module by (a). In fact, $E / E_{1}=E(M / N)$.

Using the notation of (b), note that $E_{2}$ has two partial orders. One comes from $E / E_{1}$, and one is inherited from $E$. $\left(E_{2}\right.$ is not uniquely determined by $N$, though $E_{1}$ is.) Let $P$ be the partial order of $E_{2}$ coming from $E / E_{1}$. Then $P=$ $\left\{x \in E_{2}: x+E_{1} \geq 0\right\}$ contains $E_{2} \cap E^{+}$. The following example shows that, in general, this containment is proper. Let $R=\mathbf{Z}, M=\mathbf{Z} \oplus \mathbf{Z}, E=\mathbf{Q} \oplus \mathbf{Q}, N=\mathbf{Z} \oplus$ $0, E_{1}=\mathbf{Q} \oplus 0$, and $E_{2}=(1,-1) \mathbf{Q}$. Then $P=(1,-1) \mathbf{Q}^{+}$and $E_{2} \cap E^{+}=\{0\}$. Thus $\left(E_{2}, P\right)$ is not a sublattice of $E$. If, however, $E=\operatorname{lex} E_{1}$, i.e. $M=\operatorname{lex} N$ (see 3.14), then $P=E_{2} \cap E^{+}$. For then, $x \in P$ and $x+E_{1} \geq 0$ implies $x \geq 0$. This is the case if $M$ is totally ordered.

It is easy to see that a finite direct sum of $i$-f-modules (over any po-ring) is an $i$-f-module.

Corollary 3.9. Let $R$ be an essentially positive po-ring, and let $\left\{M_{a}: a \in A\right\}$ be a collection of torsion-free f-modules. The following are equivalent.

(a) $M_{a}$ is an i-f-module for each $\alpha \in A$.

(b) $\Pi M_{a}$ is an i-f-module.

(c) $\Sigma \bigoplus M_{\alpha}$ is an i-f-module.

Proof. (b) implies (c) and (c) implies (a) by 3.8(a). Let $E_{a}=E\left(M_{\alpha}\right)$. Then (a) implies that $\Pi E_{\alpha}$ is an $i$-f-module, so $\Pi M_{\alpha}$ is an $i$-f-module, again by $3.8(a)$.

Corollary 3.10. The inverse limit of torsion-free $i-f-$ modules over an essentially positive po-ring is an i-f-module.

Proof. Let $\left(\left\{M_{\alpha}: \alpha \in A\right\},\left\{f_{\alpha \beta}: \alpha \geq \beta\right\}\right)$ be an inverse limit system of $f$-modules. Then $M=\left\{\left(m_{\alpha}\right) \in \Pi M_{\alpha}: f_{a} m_{a}=m_{\beta}\right.$ whenever $\left.\alpha \geq \beta\right\}$ is the inverse limit of this system, and $M$ is an l-submodule of $\Pi M_{\alpha}$. Thus $M$ is an $i$-f-module if each $M_{a}$ is, by 3.9 and 3.8 .

Corollary 3.11. Let $M$ be a torsion-free f-module over an essentially positive directed po-ring $R$. Then $M$ is an i-f-module if and only if every torsion-free bomomorphic image of $M$ is an i-f-module.

Proof. This follows immediately from 3.8(b).

Proposition 3.12. Let $R$ be an essentially positive po-ring with $Z(R)=0$, and let $S$ be a directed (in its canonical order) right quotient ring of $R$. Suppose that $M$ is a torsion-free f-module over $R$. Then $M$ is an i-f-module if and only if it is contained in a torsion-free i-f-module over $S$. In fact, $E\left(M_{R}\right)$ is an f-module over $S$ and an injective S-module. 
Proof. Let $E=E\left(M_{R}\right)$ be an $f$-module extension of $M$. By [23, Theorem 3 and Proposition 10], $E$ is a $Q$-module, where $Q$ is the maximal right quotient ring of $R$. If $x \wedge y=0$ in $E, q \in Q^{+}$, and $D \in R^{\nabla}$ with $q D \subseteq R$, then $x q D^{+} \subseteq E^{+}$, so $x q \in$ $E^{+}$. Also, if $d \in D^{+}$, then $(x q \wedge y) d=x q d \wedge y d=0$, so $x q \wedge y=0$. Thus $E$ is a distributive $l$-module over $Q$. Since $S$ is directed (and $E_{S}$ is torsion-free) $E_{S}$ is an $f$-modile. But any $S$-essential extension of $E$ is also an $R$-essential extension of $E$, so $E_{S}$ is injective.

Now suppose that $M$ is an $R$-submodule of a torsion-free injective $f$-module $K_{S}$. Since $E\left(K_{R}\right)$ is an $S$-module, it is an $S$-essential extension of $K$, so $K$ is $R$-injective. By $3.8, M$ is an $i$-f-module.

Theorem 3.13. A totally ordered domain has a nonzero torsion-free i-f-module if and only if it is a right Ore domain.

Proof. Let $M$ be a torsion-free $i$-f-module over the totally ordered domain $R$, and let $0<x \in M$. Then $R \rightarrow x R$ is an $f$-module homomorphism, so $R / r(x) \cong x R$. Since $M$ is torsion-free, $r(x)$ is not an essential right ideal of $R$. Let $0 \neq J$ be a right ideal such that $J \cap r(x)=0$, and take $0<y \in J$. Then $y R$ is isomorphic to an $f$-submodule of $M$, so it is an $i$-f-module. But $y R \cong R$ as $f$-modules, so $R_{R}$ is an $i$-f-module. Therefore, $E\left(R_{R}\right)$ satisfies the condition of Theorem 3.4, and so $R$ is a right Ore domain, by [1, 3.1 and 5.2].

Theorem 3.13 is not true for an arbitrary $f$-ring with $Z(R)=0$, i.e. a torsionfree $f$-ring can have a torsion-free $i$-f-module without being a $q f$-ring. In particular, let $R$ be an irredundant semiprime $f$-ring, and let $M$ be a torsion-free $f$-module over $R$. Let $R \subseteq \Pi R_{a}$ and $M \subseteq \Pi M_{a}$ be the decompositions of $R$ and $M$. Then $M$ is an $i$-f-module if and only if $M_{\alpha}=0$ for each $a$ for which $R_{a}$ is not a right Ore domain.

Proposition 3.14 (see [4, p. 239]). Let $K$ be a torsion-free f-module over an essentially positive po-ring, and let $M$ be an essential l-submodule of $K$. Suppose that $N$ is a convex l-submodule of $M$, and let $K_{1}=\mathrm{Cl}_{K} N$.

(a) If $M=\operatorname{lex} N$, then $K=\operatorname{lex} K_{1}$.

(b) If $K=\operatorname{lex} K_{1}$, then $M=\operatorname{lex} \mathrm{Cl}_{M} N$.

(c) Suppose that $K=E(M)$ and $K=$ lex $K_{1}$. If $K_{2}$ is any $R$-complement of $K_{1}$ in $K$, then $K_{2}$ is a totally ordered submodule of $K$, and

$$
K^{+}=\left\{y+z \in K_{1} \oplus K_{2}: z>0 \text {, or } z=0 \text { and } y \geq 0\right\} \text {. }
$$

Proof. (a) Suppose that $y \in K^{+\backslash} \backslash K_{1}$ and $x \in K_{1}$. Then $(y-x)^{+} \neq 0$. Assume that $(y-x)-\neq 0$, also. There exists $0 \leq d \in(M: y) \cap(M: x)=D$ such that $(y-x)-d \neq 0$. So $(y-x)^{+} D^{+} \subseteq N$, since $N$ contains all the nonunits of $M[5, \mathrm{p} .111]$. Therefore $(y-x)^{+} \in K_{1}$. Similarly, $(y-x)^{-} \in K_{1}$. Thus $y-x \in K_{1}$ and $y \in K_{1}$. So $(y-x)^{-}=0$ and $y \geq x$. Since $K_{1}$ is prime in $K$ by $2.1(\mathrm{c}), K=\operatorname{lex} K_{1}$. 
(b) If $x \in M^{+} \backslash \mathrm{Cl}_{M} N$, then $x \in K^{+} \backslash K_{1}$, since $K_{1} \cap M=\mathrm{Cl}_{M} N$. Therefore $x>$ $\mathrm{Cl}_{M} N$. Clearly, $\mathrm{Cl}_{M} N$ is prime in $M$.

(c) The remarks following 3.8 show that $K_{2}$ is totally ordered. Since $K=$ lex $K_{1}$, we clearly have $K^{+}=\left\{y+z \in K_{1} \oplus K_{2}: z>0\right.$ or $z=0$ and $\left.y \geq 0\right\}$.

We show next that the $i$-f-property is a local property.

Theorem 3.15. Suppose that $R$ is a right noetherian, essentially positive, directed po-ring, and let $M$ be a subdirect product of totally ordered torsion-free $f$-modules. Then $M$ is an i-f-module if and only if $C_{R}(g)$ is an i-f-module for each $g \in M$.

Proof. Suppose that $M$ is a subdirect product of the family $\left\{M_{a}: \alpha \in A\right\}$ of totally ordered torsion-free $f$-modules. We may assume that $M_{\alpha}=M / N_{\alpha}$ for each $\alpha$ $\epsilon A$. Therefore, if $x+N_{a} \in M_{a}$, then $C_{R}\left(x+N_{\alpha}\right)=\left(C_{R}(x)+N_{\alpha}\right) / N_{a}$. If $C_{R}(x)$ is an $i$-f-module, then so is $\left(C_{R}(x)+N_{\alpha}\right) / N_{\alpha}$, by 3.11. Now suppose that $M$ is an $i$-f-module locally, i.e. $C_{R}(g)$ is an $i$-f-module for each $g \in M$. The preceding remarks show that each $M_{a}$ is also an $i$-f-module locally. If each $M_{a}$ is an $i-f-$ module, then $M$ is an $i$-f-module by 3.9 and 3.8. So, without loss of generality, we may assume that $M$ is totally ordered.

Let $x \in E(M)=E$ and let $\left\{N_{a}: \alpha \in A\right\}=\left\{C_{R}(x d): d \in(M: x)^{+}\right\}$. If $E_{\alpha}=E\left(N_{\alpha}\right)$ $\subseteq E$, then $E_{a}$ is an $f$-module extension of $N_{a}$ by hypothesis. Since $M$ is totally ordered, $\left\{N_{a}: a \in A\right\}$ is totally ordered by inclusion, and so is the family $\left\{E_{a}: \alpha\right.$ $\epsilon A$. Thus $N=\bigcup N_{\alpha}$ is a submodule of $E_{1}=\bigcup E_{a}$. Since $R$ is noetherian, and since $E_{1}$ is the direct limit of the $E_{a}, E_{1}$ is an injective $R$-module ([3,p. 17] and $\left[10\right.$, p. 53]). Clearly, $E_{1}$ is an $f$-module extension of $N$ with positive cone $\bigcup_{E_{\alpha}^{+}}^{+}$, and it is the injective hull of $N$.

Now $x(M: x)^{+} \subseteq N \subseteq E_{1}$; so $x \in E_{1}$, since $E_{1}$ is closed in $E$. If $d_{1}, d_{2} \in R^{+}$, then $\left(x d_{1}\right)^{+} \wedge\left(x d_{2}\right)^{-}=x^{+} d_{1} \wedge x^{-} d_{2}=0$. But then $M$ is an $i-f$-module, by 3.4 .

The converse follows from 3.8 .

Corollary 3.16. Let $R$ be a right noetherian, essentially positive, directed po-ring, and let $M$ be a subdirect product of totally ordered torsion-free R-modules. If $M$ is a finitely-valued f-module, then $M$ is an i-f-module if and only if $C_{R}(g)$ is an i-f-module for every special element $g$ of $M$.

Proof. If $g \in M$, then $C_{R}(g)=C_{R}\left(g_{1}\right) \oplus \cdots \oplus C_{R}\left(g_{n}\right)$, where each $g_{i}$ is special [20]. Now use 3.14 .

Note that the proof of 3.15 is valid if, instead of assuming that $R$ is noetherian, one assumes that $M$ has the maximum condition on convex $l$-submodules.

We consider next the Hahn product of strict $i$-f-modules. Let $\Gamma$ be a rooted po-set, and suppose that for each $p \in \Gamma, M_{p}$ is an f-module over the po-ring $R$. Suppose further that $M_{p}$ is totally ordered if $p$ is not a minimal element of $\Gamma$. 
For $v \in \Pi M_{p}(R$-module product $)$ define the support (supp) of $v=\{p \in \Gamma: v(p) \neq 0\}$. It is well known $([7]$ or [24]) that

$$
V\left(\Gamma, M_{p}\right)=\left\{v \in \mathrm{II} M_{p}: \operatorname{supp} v \text { has the maximum condition }\right\}
$$

is an l-group when provided with the positive cone

$$
\dot{V}^{+}=\{v \in V: v(p)>0 \text { whenever } p \text { is a maximal element of supp } v\} \cup\{0\} \text {. }
$$

An $f$-module $M$ is called strict if it satisfies the following condition: $(x, r) \epsilon$ $M^{+} \times R^{+}$and $x r=0$ implies $x=0$ or $r=0$. Note that if $R$ has a strict $f$-module, then $R$ is a po-domain, i.e. $a, b \in R^{+}$and $a b=0$ implies $a=0$ or $b=0$.

Proposition 3.17. Suppose that $R$ is directed and each $M_{p}$ is strict. Then $V$ $=V\left(\Gamma, M_{p}\right)$ is an f-module over $R$.

Proof. Since $(v r)(p)=v(p) r$ for all $v \in \Pi M_{p}, r \in R$, and $p \in \Gamma$, supp $v r \subseteq$ $\operatorname{supp} v$. Thus $V$ is an $R$-module. If $v \in V^{+}, p$ is a maximal element of $\operatorname{supp} v$, and $0<r \in R^{+}$, then $(v r)(p)=v(p) r>0$, since $M_{p}$ is strict. So $V$ is an $l$-module over $R$. If $P$ is a maximal chain of $\Gamma$, then $V_{P}=\{v \in V: \operatorname{supp} v \subseteq P\} \cong V\left(P, M_{p}\right)$ as $l$-modules, and $V$ is a subdirect product of the $V_{P}$. Thus $V$ is an $f$-module provided each $V_{P}$ is an $f$-module. So we may assume that $\Gamma$ is totally ordered.

Let $0 \neq v \in V, 0<r \in R^{+}$, and let $q$ be the maximal element of supp $v$. Note that supp $v=\operatorname{supp} v r$, and $v(q)<0$ if and only if $(v r)(q)<0$, since $M_{q}$ is strict. Therefore, $v^{+} r=(v r)^{+}$. So $V$ is a distributive $l$-module over $R$. Hence it is an $f-$ module.

Proposition 3.18. Let $R$ be an essentially positive directed po-ring, and let $M_{p}$ be an i-f-module for each $p$ in the rooted po-set $\Gamma$. Suppose further that $M_{p}$ is totally ordered if $p$ is not a minimal element of $\Gamma$. If eacb $E_{p}=E\left(M_{p}\right)$ is strict, then $V\left(\Gamma, E_{p}\right)=E\left(V\left(\Gamma, E_{p}\right)\right)$ and $V\left(\Gamma, M_{p}\right)$ is an i-f-module.

Proof. Since $\Pi E_{p}$ is an injective module, $V\left(\Gamma, E_{p}\right) \subseteq \Pi E_{p}$ has an injective hull $E\left(V\left(\Gamma, E_{p}\right)\right)=E$ contained in $\Pi E_{p}$. Let $w \in E$ and $0 \neq d \in R^{+}$such that $w d \in V\left(\Gamma, E_{p}\right)$. Suppose that $p_{1}<p_{2}<\cdots$ is a chain in supp $w$. Since $p_{i}$ is not a minimal element of $\Gamma$ for $i>1, w\left(p_{i}\right)>0$ or $w\left(p_{i}\right)<0$. Thus $p_{i} \in \operatorname{supp} w d$ for $i>1$. This is impossible, since supp $w d$ has the maximum condition. So $w \in$ $V\left(\Gamma, E_{p}\right)$ and $E\left(V\left(\Gamma, E_{p}\right)\right)=V\left(\Gamma, E_{p}\right)$. Since $V\left(\Gamma, M_{p}\right)$ is an $l$-submodule of $V\left(\Gamma, E_{p}\right), V\left(\Gamma, M_{p}\right)$ is an $i$-f-module.

If $R$ is commutative, then $E$ is strict provided $M$ is, but we do not know if this is true for any $R$. The po-ring $R$ in 3.18 need not be a domain. Diem [8] has given the following example of a commutative $l$-domain that is not a domain: $R=$ $\mathrm{Q} a \oplus \mathbf{Q} b$ as $l$-groups and $a^{2}=b^{2}=a b=b a=a$. We remark that the essential ideals of $R$ are those ideals that contain $\mathbf{Q} a+\mathbf{Z} q b$ for some $0 \neq q \in \mathbf{Q}$. Thus $R$ 
is essentially positive. As a ring $R=\mathrm{Q} a \oplus \mathbf{Q}(a-b)$, so $\mathbf{Q} a$ is a strict $i$-f-module over $R$.

We close this section with a remark about extending homomorphisms.

Proposition 3.19. Let $N$ and $K$ be distributive l-modules over the essentially positive po-ring $R$, and suppose that $M$ is an essential l-submodule of $K$. If $f \epsilon$ $\operatorname{Hom}_{R}(M, N)$ is an l-bomomorphism and if $N$ is torsion-free, then any $g \in$ $\operatorname{Hom}_{R}(K, N)$ extending $f$ is an l-bomomorphism, and $g$ is unique.

Proof. Since $N$ is torsion-free and $M$ is essential in $K, g$ is unique. If $x \in$ $K$ and $d \in(M: x)^{+}$, then

$$
\left[g\left(x^{+}\right)\right] d=g\left(x^{+} d\right)=g\left[(x d)^{+}\right]=f\left[(x d)^{+}\right]=[f(x d)]^{+}=[g(x d)]^{+}=\left[(g x)^{+}\right] d .
$$

So $g\left(x^{+}\right)=(g x)^{+}$.

Corollary 3.20. Let $R$ be an essentially positive po-ring, and let $M$ be an i$f$-module over $R$. Then any l-bomomorphism from $M$ into a torsion-free injective $f$-module $N$ bas a unique extension to an l-bomomorphism from $E(M)$ to $N$.

4. Relative injective $f$-modules. Ribenboim [19] has observed that there are no injectives in the category of unital po-modules over a po-domain. Consequently, he defined and studied a certain type of relative injectivity. Let $\mathcal{C}$ be a category whose objects are sets, and let $\boldsymbol{\kappa}_{\alpha}$ be an infinite cardinal number. An object $E$ of $\mathcal{C}$ is called $\boldsymbol{\aleph}_{\alpha}$-injective if whenever $C$ is a subobject of $A$ in $\mathcal{C}$ and $\operatorname{card}(A)$ $<\boldsymbol{\aleph}_{\alpha}$, then every morphism $C \rightarrow E$ can be extended to a morphism $A \rightarrow E$.

Weinberg [25] has recently given a characterization of the $\boldsymbol{N}_{\alpha}$-injectives in the category of l-groups. In this section we show that his characterization (and his methods) holds in the category of torsion-free $f$-modules over an irredundant semiprime right $q f$-ring.

Definition 4.1. An l-group $M$ is an almost- $\eta_{\alpha}$-group if, for each pair of subsets $X$ and $Y$ of $M$ such that $X<Y$ and $\operatorname{card}(X \cup Y)<\boldsymbol{N}_{a}$, there exists an $a$ in $M$ such that $X \leq a \leq Y$.

Definition 4.2 (Weinberg [25]). An element $y$ in an l-group $M$ is said to split $b$ from $a$ if $y \geq a^{+}, y \wedge a^{-}=0$, and $(b-y)^{+} \wedge y=0$. M is self-splitting if each ordered pair of elements of $M$ is split by some element in $M$.

The cardinal number $\boldsymbol{K}_{a}$ is called regular provided $\bigcup\left\{X_{i}: i \in I\right\}$ has cardinality less than $\boldsymbol{\aleph}_{a}$ whenever $I$ and each $X_{i}$ have cardinality less than $\boldsymbol{\aleph}_{a}$.

Theorem 4.3 (Weinberg [25]). Let $\boldsymbol{N}_{\alpha}$ be a regular cardinal number, and let $M$ be an l-group. The following are equivalent.

(a) $M$ is $\boldsymbol{\aleph}_{\alpha}$-injective.

(b) $M$ is a divisible, self-splitting, almost- $\eta_{\alpha}-$ group in which any two pairwise disjoint subsets of cardinality less than $\boldsymbol{\aleph}_{a}$ bave disjoint upper bounds. 
Now let $M_{R}$ be an $f$-module. We will show, with not too many restrictions on $R$, that Weinberg's four conditions are necessary for $M$ to be an $\boldsymbol{\aleph}_{a}$-injective $f-$ module. The converse is much harder and we only have it for the case that $R$ is an irredundant semiprime $q f$-ring.

Lemma 4.4. Let $M$ be a torsion-free i-f-module over the directed po-ring $R$, let $\mathcal{C}$ be the category of torsion-free f-modules over $R$. If $M$ is $\boldsymbol{N}_{\alpha}$-injective in $\mathcal{C}$, then so is $E=E\left(M_{R}\right)$.

Proof. Suppose that $N \in \mathcal{C}$ with $\operatorname{card}(N)<\boldsymbol{N}_{\alpha}$. Let $N^{\prime}$ be an l-submodule of $N$, and let $f$ be a homomorphism from $N^{\prime}$ into $E$. Then $N^{\prime \prime}=f^{-1}(M)$ is an essential $l$-submodule of $N^{\prime}$. We have the following diagram:

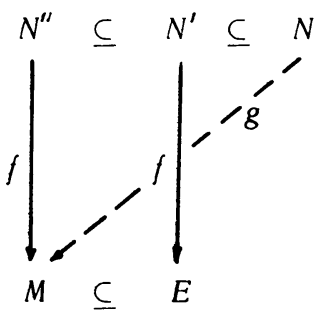

where $g$ comes from the fact that $M$ is $\boldsymbol{\aleph}_{\alpha}$-injective. Since $N^{\prime \prime} \subseteq \operatorname{ker}(g-f)$, since $N^{\prime \prime}$ is essential in $N^{\prime}$, and since $M$ is torsion-free, $N^{\prime} \subseteq \operatorname{ker}(g-f)$. Thus $f\left(N^{\prime}\right) \subseteq$ $M$, and $g$ is an extension of $f$.

Corollary 4.5. Let $M$ be a torsion-free i-f-module over the directed po-ring $R$. If $\boldsymbol{K}_{\alpha}>\operatorname{card}(R)$ and if $M$ is $\boldsymbol{K}_{\alpha}$-injective in the category of torsion-free f-modules over $R$, then $M=E\left(M_{R}\right)$.

Proof. Take $x \in E$ and let $N_{1}=x R_{*}$. It is known that the sublattice of $E$ generated by $N_{1}$ is a subgroup, and is given by

$$
L\left(N_{1}\right)=\left\{\bigvee_{i=1}^{m} \bigwedge_{i=1}^{n} s_{i j}: s_{i j} \in N_{1}\right\} .
$$

If $r \in R^{+}$, then $L\left(N_{1}\right) r \subseteq L\left(N_{1}\right)$, since $E$ is an $f$-module. Thus $L\left(N_{1}\right)$ is an $l$-submodule of $E$, since $R$ is directed. Clearly, $\operatorname{card}\left(L\left(N_{1}\right)\right)<\boldsymbol{N}_{a}$. In the proof of 4.4, let $N^{\prime}=N=L\left(N_{1}\right), N^{\prime \prime}=L\left(N_{1}\right) \cap M$, and let $f$ be the inclusion map from $N^{\prime}$ to E. Then $f\left(L\left(N_{1}\right)\right) \subseteq M$, so $x \in M$.

Lemma 4.6. Let $R$ be a directed po-domain which bas a strict totally ordered module $K$. Then any f-module $M$ can be embedded in an f-module which contains, for each $a$ in $M$, an element $y$ that splits $b$ from a for every $b$ in $M$.

Proof. $M$ can be embedded in a product of a family of totally ordered modules $\left\{M_{i}: i \in I\right\}$. Let $N_{i}=M \oplus K$ as $R$-modules with positive cone defined by $N_{i}^{+}=$ $\{(m, k): k>0$, or $k=0$ and $m \geq 0\}$. Define $y \in \Pi N_{i}$ by $y(i)=0$ if $a^{+}(i)=0$, and 
$y(i)=\left(0, k_{0}\right)$ if $a^{+}(i)>0$, where $k_{0}$ is a fixed nonzero positive element of $K$.

Proposition 4.7. Let $R$ be a directed po-domain which bas a strict totally ordered module $M$, and let $\boldsymbol{\aleph}_{a}$ be a cardinal number greater than $\operatorname{card}(R)$. If $M$ is an $\boldsymbol{\aleph}_{a}$-injective f-module, then $M$ is self-splitting and each pair of pairwise disjoint subsets of $M$ of cardinality less than $\boldsymbol{\aleph}_{a}$ bave disjoint upper bounds.

Proof. Let $a, b \in M$, and let $A$ be the l-submodule of $M$ generated by $a$ and b. By 4.6, $A$ is imbeddable in an $f$-module $C$ containing an element $y$ which splits $b$ from $a$. Let $B$ be the $l$-submodule of $C$ generated by $A$ and $y$. Then $\operatorname{card}(B)<$ $\boldsymbol{\aleph}_{\alpha}$, and the injection of $A$ into $M$ can be extended to a homomorphism $\phi$ from $B$ into $M$, since $M$ is $\aleph_{a}$-injective. Clearly, $\phi(y)$ splits $b$ from $a$. Thus $M$ is selfsplitting.

Let $A_{1}$ and $A_{2}$ be pairwise disjoint subsets of $M$ such that $\operatorname{card}\left(A_{1}\right)+$ $\operatorname{card}\left(A_{2}\right)<\boldsymbol{N}_{\alpha}$. Embed $M$ in a product of totally ordered $R$-modules $M \rightarrow \Pi M_{i}$. As in the proof of 4.6 , let $N_{i}$ be the lexicographic extension of $M_{i}$ by $K$. Define $t_{j}$ in $N=\Pi N_{i}$, for $j=1,2$, by $t_{j}(i)=\left(0, k_{0}\right)$ if $\pi_{i}\left(A_{j}\right) \neq 0$ and $t_{j}(i)=0$ if $\pi_{i}\left(A_{j}\right)=$ 0 . Then $t_{1} \wedge t_{2}=0$ and $t_{j} \geq A_{j}$. Let $L$ be the $l$-submodule of $N$ generated by $A_{1}$ $\cup A_{2}$, and let $P$ be the $l$-submodule of $N$ generated by $L$ and $\left\{t_{1}, t_{2}\right\}$. Then $\operatorname{card}(P)<\boldsymbol{N}_{a}$, and the injection of $L$ into $M$ can be extended to a homomorphism $\phi$ from $P$ into $M$. Thus $\phi\left(t_{1}\right) \wedge \phi\left(t_{2}\right)=0$, and $\phi\left(t_{j}\right) \geq A_{j}$.

A totally ordered set $T$ is said to be dense if for all $a<b$ in $T$ there exists $x \in T$ such that $a<x<b$. If $M$ is a totally ordered module over a po-ring $R$, then $\mathbf{Q} \otimes_{Z} M$ is a dense totally ordered $R$-module containing $M$.

Lemma 4.8. Let $M$ be a totally ordered module over the po-ring $R$, and suppose that $X$ and $Y$ are subsets of $M$ such that $X<Y$. Then $M$ can be embedded in $a$ totally ordered module $N$ containing an element a satisfying $X<a<Y$. Moreover, if $R$ is right noetherian and $M$ is torsion-free, then such an $N$ may be found which is torsion-free.

Proof. By the preceding remark we may assume that $M$ is dense. Suppose that no element of $M$ lies between $X$ and $Y$. Then either $X$ is nonempty and has no last element or $Y$ is nonempty and has no first element. Suppose, for example, that $X$ is nonempty and has no last element. For $x \in X$, let $A_{x}=\{z \in X: z>x\}$. Since $X$ has no last element, $\left\{A_{x}: x \in X\right\}$ has the finite intersection property. Therefore, there is an ultrafilter $\mathcal{E}$ on $X$ containing $\left\{A_{x}: x \in X\right\}$.

For each $b \in M^{X}$ let $Z(b)=\{x \in X: b(x)=0\}$. Let $I=\left\{f \in M^{X}: Z(f) \in \mathcal{E}\right\}$. Then $I$ is a prime submodule of $M^{X}$. Embed $M$ in $M^{X} / I$ via the map $m \rightarrow\langle m\rangle+$ $I$, where $\langle m\rangle(x)=m$ for all $x \in X$. If $e \in M^{X}$ is the identity on $X$, i.e. $e(x)=x$ for all $x \in X$, then $\langle X\rangle+I\langle e<\langle Y\rangle+I$.

Suppose that $M$ is torsion-free and $R$ is right noetherian. Let $f \in M^{X}$ and $D$ $\in R^{\nabla}$ such that $f d \subseteq I$. Since $R$ is noetherian, $D=d_{1} R_{*}+\cdots+d_{n} R_{*}$. Clearly, 
$Z(f) \subseteq \bigcap\left\{Z\left(f d_{i}\right): i=1, \cdots, n\right\}$. Conversely, if $\left(f d_{i}\right)(x)=0$ for $1 \leq i \leq n$, then $f(x) D$ $=0$. Thus $f(x)=0$, since $M$ is torsion-free. So $Z(f)=\bigcap\left\{Z\left(f d_{i}\right): i=1, \cdots, n\right\}$. Hence $Z(f) \in \mathcal{E}$ and $f \in I$.

Proposition 4.9. Let $M$ be an $\boldsymbol{N}_{a}$-injective f-module over the directed po-ring $R$, and suppose $\boldsymbol{K}_{\alpha}>\operatorname{card}(R)$. Then $M$ is an almost- $\eta_{\alpha}-$ module.

Proof. Suppose that $X$ and $Y$ are subsets of $M$, each of which has cardinality less than $\boldsymbol{K}_{\alpha}$, and $X<Y$. Assume $M$ is embedded in the product $\Pi M_{j}$, where each $M_{j}$ is totally ordered. Let $N_{j}$ be a totally ordered module containing $M_{j}$ and an element $t_{j}$ such that $\pi_{j}(X) \leq t_{j} \leq \pi_{j}(Y)$. Let $t \in \Pi N_{j}=K$ be defined by $t(j)=t_{j}$. Finally, let $A$ be the $l$-submodule of $M$ generated by $X \cup Y$, and let $B$ be the $l$ submodule of $K$ generated by $A$ and $t$. Then $\operatorname{card}(B)<\boldsymbol{\aleph}_{a}$, and the injection of $A$ into $M$ can be extended to $B$. Thus the image of $t$ in $M$ lies between $X$ and $Y$.

Suppose that $R$ is right noetherian (and directed), and $M$ is a subdirect product of totally ordered torsion-free $R$-modules. If $M$ is $\boldsymbol{\aleph}_{\alpha}$-injective in the category of torsion-free $f$-modules over $R$, then $M$ is an almost- $\eta_{a}$-module. The proof is the same as that of 4.9. Of course, if $M$ is a torsion-free $f$-module over an essentially positive po-ring $R$, then $M$ is $\boldsymbol{N}_{a}$-injective in the category of $R$-f-modules if and only if it is $\boldsymbol{\aleph}_{a}$-injective in the category of torsion-free $R$-f-modules. For then the torsion submodule, $\mathrm{ClCl}_{A} 0=Z_{2}(A)$, of each $f$-module $A$ is a convex $l$ submodule; so an $f$-module homomorphism $A \rightarrow M$ induces a torsion-free $f$-module homomorphism $A / Z_{2}(A) \rightarrow M$.

For the remainder of this section $\aleph_{a}$ will be a regular cardinal number.

Theorem 4.11. Let $R$ be a totally ordered right Ore domain, and let $M$ be a torsion-free f-module over $R$. If $\boldsymbol{\aleph}_{\alpha}>\operatorname{card}(R)$, then $M$ is $\boldsymbol{\aleph}_{\alpha}$-injective in the category of torsion-free f-modules over $R$ if and only if it is injective in the category of $R$-modules and $\boldsymbol{N}_{a}$-injective in the category of l-groups.

Proof. If $M$ is $\aleph_{a}$-injective, then $4.5,4.7,4.9$, and 4.3 imply that $M_{R}$ is injective and that $M$ is an $\boldsymbol{K}_{\alpha}$-injective $l$-group.

Conversely, if $M_{R}$ is torsion-free and injective, then $M$ is a vector lattice over $D$, where $D$ is the totally ordered right quotient division ring of $R$ (see 3.12). Thus we may assume that $R=D$. Now copy the proof of 4.3 given in [24] for the case that $R=\mathbf{Q}$.

Corollary 4.12. Let $R$ be an irredundant semiprime right qf-ring, and let $M$ be a torsion-free f-module over $R$. Suppose that $\boldsymbol{K}_{a}>\operatorname{card}(R)$. Then $M$ is $\boldsymbol{\aleph}_{a}$-injective in the category of (torsion-free) f-modules over $R$ if and only if $M$ is an injective $R$-module and an $\boldsymbol{N}_{\alpha}$-injective l-group.

Proof. Let $R \subseteq \Pi R_{\lambda} \subseteq \Pi D_{\lambda}=Q$ be the decomposition of $R$ (see 2.7). If $M$ is either $\aleph_{\alpha}$-injective or $R$-injective, then $M=\Pi E_{\lambda}$, where $E_{\lambda}$ is an f-module over 
$R\left(R_{\lambda}\right)$ and an injective $R-\left(R_{\lambda^{-}}\right)$module $(3.6,4.5$, and the proof of 2.9). By the usual argument, it is easily seen that $M$ is $\boldsymbol{\aleph}_{\alpha}$-injective if and only if each $E_{\lambda}$ is $\boldsymbol{\aleph}_{\alpha}$-injective.

Now $E_{\lambda}$ is an $\boldsymbol{\aleph}_{\alpha}$-injective $f$-module over $R$ if and only if it is an $\boldsymbol{\aleph}_{a}$-injective $f$-module over $R_{\lambda}$. For suppose that $E_{\lambda}$ is $\aleph_{a}$-injective with respect to $R$. Let $A$ be an $f$-submodule of the $R_{\lambda^{-}} f$-module $B\left(\operatorname{card}(B)<\boldsymbol{\aleph}_{a}\right)$, and let $f: A \rightarrow E$ be a map in the category of $R_{\lambda}$-f-modules. Since $R_{\lambda}$ is a homomorphic image of $R, A$ and $B$ are naturally $f$-modules over $R$, and then $f$ is an $R$-homomorphism. Let $g$ : $B \rightarrow E_{\lambda}$ be an $R$-extension of $f$. Then $g$ is clearly an $R_{\lambda}$-extension of $f$; so $E_{\lambda}$ is $\aleph_{\alpha}$-injective over $R_{\lambda}$.

On the other hand, suppose that $E_{\lambda}$ is $\boldsymbol{\aleph}_{\alpha}$-injective over $R_{\lambda}$. Let $A$ be an $f-$ submodule of the torsion-free $R$-f-module $B\left(\operatorname{card}(B)<\boldsymbol{\aleph}_{\alpha}\right)$, and let $f: A \rightarrow E$ be a map in the category of $R$-f-modules. By 3.20, we may assume that $A$ and $B$ are injective $R$-modules. Thus $A$ and $B$ (and $E_{\lambda}$ ) are $Q$-modules (see 3.12), and $f$ is a $Q$-map. Let $g: B \rightarrow E_{\lambda}$ be the $R_{\lambda}$-extension of $f$. Then $g$ is, in fact, a $Q$-extension of $f$, hence an $R$-extension. So $E_{\lambda}$ is $\boldsymbol{N}_{\alpha}$-injective over $R$.

In summary, we have $M$ is $R$ - $\mathcal{N}_{\alpha}$-injective if and only if $M=\Pi E_{\lambda}$, where $E_{\lambda}$ is $R_{\lambda}$ - $\boldsymbol{N}_{\alpha}$-injective. But $E_{\lambda}$ is $R_{\lambda}-\boldsymbol{N}_{\alpha}$-injective if and only if it is $\mathbf{Z}$ - $\boldsymbol{K}_{\alpha}$-injective, by Theorem 4.11. Thus $M$ is $R-\boldsymbol{N}_{\alpha}$-injective exactly when it is an injective $R$-module and an $\boldsymbol{K}_{a}$-injective $l$-group.

Note that there are no injectives in the category of torsion-free $R$-f-modules. For any nonzero torsion-free injective $R$ - $f$-module gives rise to a nonzero torsionfree injective $R_{\lambda}$-f-module for some $\lambda$, and there are none. There are quasi-injectives, however. In particular, if $R$ is any semiprime qf-ring and $f$ is a homomorphism from the $l$-submodule $M$ of $Q_{R}$ into $Q_{R}$, then $f$ may be extended to $E(M) \subseteq$ $Q$, by 3.20 , and thus it can be extended to $Q$, since $E(M)$ is a summand of $Q$. So $Q_{R}$ is a quasi-injective $R$ - $f$-module.

The next corollary is an immediate consequence of Weinberg's theorem (Theorem 4.3) and 4.12.

Corollary 4.13. The following statements are equivalent for a torsion-free $f-$ module $M$ over an irredundant semiprime right qf-ring $R\left(\operatorname{card}(R)<\boldsymbol{N}_{a}\right)$.

(a) $M$ is $\boldsymbol{N}_{a}$-injective.

(b) $M$ is an injective $R$-module, and a self-splitting almost- $\eta_{a}$-module in which any two pairwise disjoint subsets of cardinality less than $\boldsymbol{N}_{\alpha}$ bave disjoint upper bounds.

Finally, we remark that there are enough $\boldsymbol{K}_{\alpha}$-injectives for embedding purposes when $R$ is an irredundant semiprime right $q f$-ring. The proof is the same as that given by Weinberg in [25] for $R=\mathbf{Z}$. We can reduce to the case where $R$ is a totally ordered division ring. Then the idea is to enlarge the given vector lattice $M$, 
successively, via vector lattices in which every pair of elements of $M$ is split, every pair of disjoint subsets of $M$ of small cardinality have disjoint upper bounds, and in which there is an element between every pair of subsets of $M$ of small cardinality, one of which is smaller than the other. By repeating this procedure inductively one eventually gets a vector lattice having the properties of $4.3(\mathrm{~b})$.

5. Remarks on an Hahn embedding theorem for $f$-modules. Let $M$ be a torsionfree $f$-module over the irredundant semiprime right $q f$-ring $R$, and let $R \subseteq \Pi R_{\lambda} \subseteq$ $\Pi D_{\lambda}=Q$ and $M \subseteq \Pi M_{\lambda} \subseteq \Pi E_{\lambda}=E$ be the representations of $R$ and $M$, respectively. Let $\Gamma_{\lambda}$ be the $D_{\lambda}$-value set of $E_{\lambda}$. For each lower submodule $M_{a} \in \Gamma_{\lambda}$, let $M^{\alpha}$ be the convex $l$-submodule of $E_{\lambda}$ that covers it. Since the proof of the Hahn embedding theorem for l-groups [7] is valid for a vector lattice over a totally ordered division ring, $E_{\lambda}$ is $D_{\lambda}$-value embedded in the Hahn product $V_{\lambda}=V\left(\Gamma_{\lambda}, M^{\alpha} / M_{\alpha}\right)$. Thus the $f$-module $M_{R}$ is embedded in the product of the $V_{\lambda}$. Let $\Gamma$ be the cardinal sum of the $\Gamma_{\lambda}$. Then the map $\left(v_{\lambda}\right) \rightarrow \bar{v}, \bar{v}(\alpha)=v_{\lambda}(\alpha)$ embeds the $Q$-f-module II $V_{\lambda}$ onto the Hahn product $V\left(\Gamma, M^{a} / M_{a}\right)=V$. ( $V$ is a Hahn product as a $Q-f$ module, i.e. $V^{+} Q^{+} \subseteq V^{+}$: Suppose $0<v \in V$ and $0<q=\left(q_{\lambda}\right) \in Q$. Let $\alpha$ be a maximal element in the support of $v q, \alpha \in \Gamma_{\mu}$. Then $\alpha$ is a maximal element in the support of $v$, and $(v q)(\alpha)=v(\alpha)\left(q_{\lambda}\right)=v(\alpha) q_{\mu}>0$.)

If $M$ has only a finite number of nonzero components, in particular, if $R$ is a semiprime right Goldie $f$-ring, then $\Gamma$ is the $Q$-value set of $E(M)$, but in general $\Gamma$ is only contained in the latter. If a component $R_{\lambda}$ of $R$ is archimedean, in particular, if $R$ is archimedean, then the $M^{\alpha} / M_{\alpha}$ for $\alpha \in \Gamma_{\lambda}$ are $D_{\lambda}$-submodules of the reals. In this case $\Gamma_{\lambda}$ is isomorphic to the value set of $M_{\lambda}$.

\section{REFERENCES}

1. F. W. Anderson, Lattice-ordered rings of quotients, Canad. J. Math. 17 (1965), 434-448. MR 30 \#4801.

2. G. Birkhoff and R. S. Pierce, Lattice-ordered rings, An. Acad. Brasil. Ci. 28 (1956), 41-69. MR 18, 191.

3. H. Cartan and S. Eilenberg, Homological algebra, Princeton Univ. Press, Princeton, N. J., 1956. MR 17, 1040.

4. P. F. Conrad, Some structure theorems for lattice-ordered groups, Trans. Amer. Math. Soc. 99 (1961), 212-240. MR 22 \#12143.

5. The lattice of all convex l-subgroups of a lattice-ordered group, Czechoslovak Math. J. 15 (90) (1965), 101-123. MR 30 \#3926.

6. P. F. Conrad and J. E. Diem, The ring of polar preserving endomorphisms of an abelian lattice-ordered group, Illinois J. Math. 15 (1971), 222-240.

7. P. F. Conrad, J. Harvey and C. Holland, The Hahn embedding theorem for abelian lattice-ordered groups, Trans. Amer. Math. Soc. 108 (1963), 143-169. MR 27 \#1519.

8. J. E. Diem, A radical for lattice-ordered rings, Pacific J. Math. 25 (1968), 71-82. MR $37 \# 2653$.

9. B. Eckmann and A. Schopf, Über injektive Moduln, Arch. Math. (Hochschild) 4 (1953), 75-78. MR 15, 5.

10. C. Faith, Lectures on injective modules and quotient rings, Lecture Notes in Math., no. 49, Springer-Verlag, New York, 1967. MR 37 \#2791. 
11. L. Fuchs, Teilweise geordnete algebraische Strukturen, Vandenhoeck and Ruprecht in Gottingen, 1966. MR 34 \#4386.

12. A. W. Goldie, Rings with maximum condition, Lecture Notes, Yale University, New Haven, Conn., 1964.

13. - Torsion-free modules and rings, J. Algebra 1 (1964), 268-287. MR 29 \#2282.

14. P. Jaffard, Les systèmes d'idéaux, Travaux et Recherches Mathématiques, 4, Dunod, Paris, 1960. MR 22 \#5628.

15. D. G. Johnson, A structure theory for a class of lattice-ordered rings, Acta Math. 104 (1960), 163-215. MR 23 \#A2447.

16. D. G. Johnson and J. Kist, Prime ideals in vector lattices, Canad. J. Math. 14 (1962), 517-528. MR $25 \# 2010$.

17. R. E. Johnson, The extended centralizer of a ring over a module, Proc. Amer. Math. Soc. 2 (1951), 891-895. MR 13, 618.

18. L. Levy, Unique subdirect sums of prime rings, Trans. Amer. Math. Soc. 106 (1963), 64-76. MR $26 \# 136$.

19. P. Ribenboim, On ordered modules, J. Reine Angew. Math. 225 (1967), 120-146. MR 34 \#5877.

20. S. A. Steinberg, Finitely-valued f-modules, Pacific J. Math. (to appear).

21. Lattice-ordered rings and modules, Dissertation, University of Illinois, Urbana, Ill., 1970.

22. Y. Utumi, On quotient rings, Osaka Math. J. 8 (1956), 1-18. MR 18, 7.

23. Diana Yun-dee Wei, On the concept of torsion and divisibility for general rings, Illinois J. Math. 13 (1969), 414-431. MR 9, 280.

24. E. C. Weinberg, Lectures on ordered groups and rings, Lecture Notes, University of Illinois, Urbana, Ill., 1968.

25. - Relative injectives and universals for categories of ordered structures, Trans. Amer. Math. Soc. (to appear).

DEPARTMENT OF MATHEMATICS, UNIVERSITY OF MISSOURI, ST. LOUIS, MISSOURI 63121

Current address: Department of Mathematics, University of Toledo, Toledo, Ohio 43606 